Published in "Diagnostic Microbiology and Infectious Disease 95(1): 1-4, 2019"

which should be cited to refer to this work.

\title{
A selective culture medium for screening linezolid-resistant gram- positive bacteria
}

\author{
Patrice Nordmann ${ }^{\text {a,b,c,d,* }}$, Angel Rodríguez-Villodres ${ }^{\text {a,e,f }}$, Laurent Poirel a,b,c \\ a Medical and Molecular Microbiology Unit, Department of Medicine, Faculty of Science, University of Fribourg, Fribourg, Switzerland \\ b INSERM European Unit (IAME, France), University of Fribourg \\ c Swiss National Reference Center for Emerging Antibiotic Resistance (NARA), Fribourg, Switzerland \\ ${ }^{\mathrm{d}}$ Institute for Microbiology, University of Lausanne and University Hospital Centre, Lausanne, Switzerland \\ e Clinical Unit of Infectious Diseases, Microbiology and Preventive Medicine, University Hospital Virgen del Rocío, Seville, Spain \\ ${ }^{\mathrm{f}}$ Institute of Biomedicine of Seville (IBiS), University Hospital Virgen del Rocio/CSIC/University of Seville, Seville, Spain
}

Keywords:

Linezolid

Antibiotic

Screening

Enterococci

Staphylococci

\section{A B S T R A C T}

The SuperLinezolid medium was developed for screening resistance to linezolid (LZD) in Gram-positive bacteria (Staphylococcus spp., Enterococcus spp.). It was evaluated using LZD-susceptible $(\mathrm{n}=20)$ and LZD-resistant ( $\mathrm{n}=$ 17) Gram-positive isolates. The sensitivity was found to be $82 \%$ at $24 \mathrm{~h}$ ( 3 out of 17 isolates being missed), and reached $100 \%$ at $48 \mathrm{~h}$. At 48 h, a single LZD-susceptible isolate grew (specificity 95\%). By testing stools spiked with LZD-resistant Gram-positive strains, an excellent performance of the medium was observed, with a lowest detection limit ranging from $10^{1}$ to $10^{2} \mathrm{CFU} / \mathrm{ml}$. Overall, this medium is accurate for detection of LZD-resistant Gram-positive isolates after $24 \mathrm{~h}$ of culture.
Oxazolidinone antibiotics such as linezolid (LZD) are increasingly used as a consequence of an increased rate of multidrug-resistant Gram-positive pathogens (Leach et al., 2011). Considering the wide diffusion of methicillin-resistant Staphylococcus aureus and Staphylococcus epidermidis isolates worldwide (Purrello et al., 2016), as well as the wide diffusion of vancomycin-resistant Enterococcus faecium and Enterococcus faecalis (Zahedi Bialvaei et al., 2017), there is a significant need to rely on the use of LZD to treat infections caused by those multidrug-resistant isolates (Cattoir and Giard, 2014). Therefore, it not surprising that occurrence of LZD-resistant isolates is now increasingly reported, including in S. aureus, S. epidermidis, E. faecium, and E. faecalis (Bi et al., 2017; Bourgeois-Nicolaos et al., 2014; Gu et al., 2013). Consequently, accurate and rapid identification of LZD-resistant isolates is needed.

The main mechanism of resistance to LZD in Gram-positive bacteria corresponds to a specific mutation (G2576 T) in the 23S rRNA gene, preventing the binding of the drug to its target, i.e. the ribosome (Sadowy et al., 2018). Some other mutations have been reported in the 23S rRNA gene, leading to modifications of the L3, L4, and L22 ribosomal proteins, but they are much less frequent (Bi et al., 2017; Gu et al., 2013; Pfaller et al., 2017a, 2017b).

\footnotetext{
* Corresponding author. Tel.: +41-26-300-9583.

E-mail address: patrice.nordmann@unifr.ch (P. Nordmann).
}

In addition, acquired transferable LZD resistance genes have been reported, namely the $c f r, c f r(B), c f r(C)$, optrA, and poxtA genes (Sadowy, 2018). The $c f r$ gene encodes a methylase modifying the $C-8$ position of A2503 residue in the 23S rRNA methylases (Kehrenberg et al., 2005; Schwarz et al., 2000), and confers resistance not only to LZD but also to phenicols, lincosamides, pleuromutilins, and streptogramins (so called $\mathrm{PhLOPS}_{\mathrm{A}}$ resistance phenotype), but noticeably spares the novel oxazolidinone tedizolid (Long et al., 2006). This gene has been identified in S. aureus, enterococci, Streptococcus suis, and Bacillus spp., but also in several Gram-negative bacteria such as Escherichia coli and Proteus vulgaris. The $c f r(\mathrm{~B})$ gene sharing $72 \%$ nucleotide identity with $c f r$ was found in S. aureus, also conferring a $\mathrm{PhLOPS}_{\mathrm{A}}$ resistance phenotype (Marín et al., 2015). The $c f r(C)$ gene encoding a protein sharing ca. $55 \%$ amino acid identity with $\mathrm{Cfr}(\mathrm{A})$ and $\mathrm{Cfr}(\mathrm{B})$ was found in Campylobacter spp. (Tang et al., 2017).

In addition to those Cfr-like 23S rRNA methylases, another acquired resistance trait to LZD has been reported, being OptrA (Wang et al., 2015). It belongs to the ABC-F family of ATP-binding proteins, and has been characterized as a ribosomal protection protein. The corresponding gene was first identified in E. faecalis, and later in Staphylococcus sciuri and Streptococcus gallotycus (Sharkey et al., 2016).

Finally, another ribosomal protection protein, PoxtA, was recently identified from a human methicillin-resistant S. aureus (MRSA) clinical isolate isolate, and further identified in E. faecalis and E. faecium of 
Table 1

Preparation of the SuperLinezolid medium.

\begin{tabular}{lll}
\hline Compounds & Stock solution & Quantity or volume to add $^{\text {a }}$ \\
\hline BHI agar powder & - & $14.8 \mathrm{~g}$ \\
Distilled water & - & $400 \mathrm{ml}$ \\
Linezolid & $2 \mathrm{mg} / \mathrm{ml}$ & $300 \mu \mathrm{ll}$ \\
Aztreonam & $10 \mathrm{mg} / \mathrm{ml}$ & $80 \mu \mathrm{l}$ \\
Colistin sulfate & $15 \mathrm{mg} / \mathrm{ml}$ in water in glass tubes & $400 \mu \mathrm{l}$ \\
Amphotericin B & $20 \mathrm{mg} / \mathrm{ml} \mathrm{in} \mathrm{D(+)-glucose} 10 \%$ & $100 \mu \mathrm{l}$ \\
\hline
\end{tabular}

a The volume of $400 \mathrm{ml}$ of SuperLinezolid medium was for twenty plates.

animal origin (Antonelli et al., 2018). That resistance mechanism confers reduced susceptibility not only to oxazolidinone, but also to tetracyclines and phenicols.

Taking in account the potential clinical threat represented by a diffusion of LZD-resistant strains, our aim was to develop a selective culture medium for screening of LZD-resistant bacteria both among human and animal isolates.

\section{Material and methods}

\subsection{Preparation of the SuperLinezolid medium}

The necessity to prevent contamination by Gram negatives and fungi - was taken in account for the development of this medium. Based on our own experience of developing screening media (Nordmann et al., 2012, 2016), the optimal medium retained was based on the Brain Heart Infusion (BHI) medium (ref 3,564,014; Bio-Rad, Cressier, Switzerland). This medium was rich enough to enhance the growth of the Gram-positive isolates we tested.

To determine the optimal concentrations of each compound of the SuperLinezolid medium, a series of different preliminary tests was performed, using six linezolid-resistant and two linezolid-susceptible isolates, including Staphylococci and Enterococci strains. Using an inoculum with an optical density of 0.5 Mac Farland (inoculum of $\sim 10^{8} \mathrm{CFU} /$ $\mathrm{ml}$ ), a 1000 -fold dilution of the strains to be tested was made in normal saline solution and a $100-\mu \mathrm{l}$ volume was plated onto the SuperLinezolid medium. To quantify the viable bacteria in each dilution, BHI agar medium was inoculated concomitantly with $100 \mu \mathrm{l}$ of suspension and incubated overnight at $37^{\circ} \mathrm{C}$. A range of concentrations varying from 0.25 , $0.5,1,1.5,2,2.5,3,3.5$, and $4 \mathrm{mg} / \mathrm{L}$ was tested, and the $1.5 \mathrm{mg} / \mathrm{L}$ concentration was retained since it allowed to obtain the optimal sensitivity and specificity values. Colistin sulfate (tablets, MAST DIAGNOSTICS, Merseyside, UK) was added in the medium at a final concentration of $15 \mu \mathrm{g} / \mathrm{ml}$ to prevent the growth of colistin- susceptible Gram-negative isolates. In addition, considering that growth of colistin-resistant Gram-negative strains might be a source of contamination (particularly with species being intrinsically resistant or heteroresistant to colistin such as Proteus spp., Serratia spp., Hafnia spp., or Enterobacter cloacae) (Poirel et al., 2017), aztreonam was added at a concentration of $2 \mu \mathrm{g} / \mathrm{ml}$. While addition of aztreonam contributed to inhibit growth of Gramnegative bacteria (unless they produced broad-spectrum ß-lactamases), it did not modify the growth of the Gram-positive bacteria tested, including LZD-susceptible and -resistant isolates. Amphotericin B (Bristol-Myers-Squibb, Rueil-Malmaison, France) was added as an antifungi molecule at a final concentration of $5 \mu \mathrm{g} / \mathrm{ml}$. Cultures were incubated at $37^{\circ} \mathrm{C}$ during $18 \mathrm{~h}$. When no growth was observed after $24 \mathrm{~h}$, the incubation period was extended to $48 \mathrm{~h}$ to definitely assess that no growth actually occurred.

The instructions for the preparation of SuperLinezolid medium are indicated in Table 1 . The stock solutions may be kept at $-20^{\circ} \mathrm{C}$. For preparing the SuperLinezolid medium, the diluted powder of BHI was autoclaved at $121^{\circ} \mathrm{C}$ for $15 \mathrm{~min}$. After cooling this medium for one hour at $56^{\circ} \mathrm{C}$, the antibiotic stock solutions were added (Table 1). Once poured, the plates were stored at $4^{\circ} \mathrm{C}$ and protected from direct light exposure. The SuperLinezolid plates were kept one month and no major effect on its performances was noticed over time (data not shown). (See Fig. 1.)

A total of thirty-seven isolates of various Gram-positive species (Enteroccocus faecium, Enterococcus faecalis, Enterococcus casseliflavus, Staphylococcus aureus, Staphylococcus epidermidis, Staphylococcus capitis) recovered in France were tested to evaluate the performance of the SuperLinezolid medium. Seventeen of those isolates were resistant to LZD, and twenty isolates were susceptible. In addition, a total of eleven Gram-negative isolates were tested, including Enterobacteriaceae $(\mathrm{n}=9)$, Pseudomonas aeruginosa $(\mathrm{n}=1)$, and Acinetobacter baumannii $(\mathrm{n}=1)$ isolates. All those isolates were clonally-unrelated (data not shown).

The lowest limit of detection with the SuperLinezolid medium was determined for all the tested strains. Using an inoculum with an optical density of $0.5 \mathrm{McFarland}$ standard (inoculum of $\sim 10^{8} \mathrm{CFU} / \mathrm{ml}$ ), serial 10 -fold dilutions of the isolates were made in normal saline, and $100-\mu \mathrm{l}$ portions were plated onto the SuperLinezolid medium. To quantify the viable

A

B

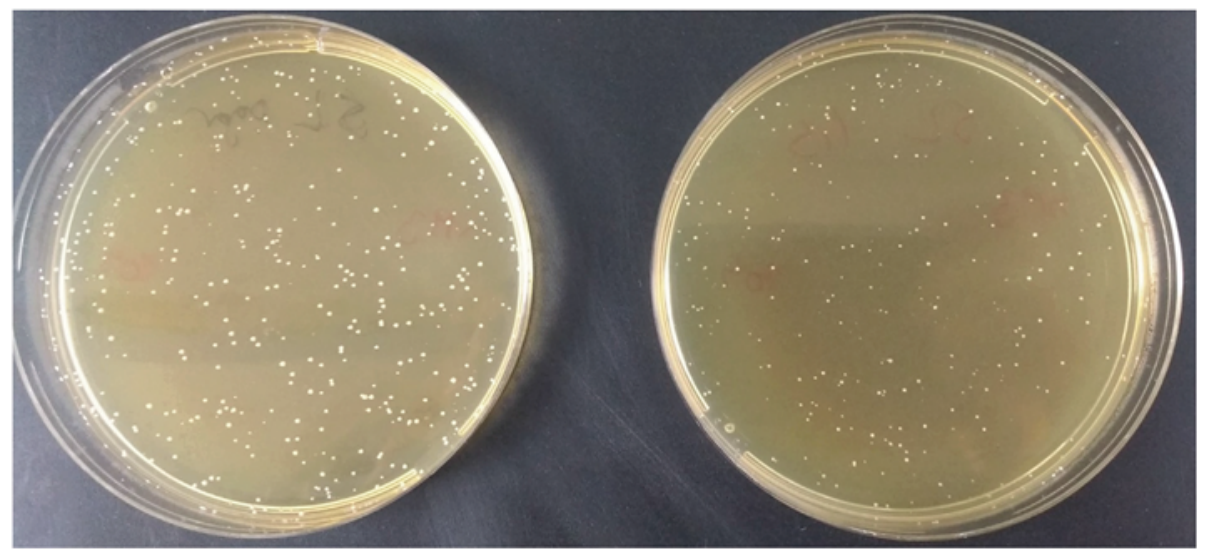

Fig. 1. LZD-resistant S. epidermidis (A), and E. faecium (B) growing onto the SuperLinezolid medium. 
Table 2

Lowest limits of detection of the SuperLinezolid medium for linezolid-resistant isolates.

\begin{tabular}{|c|c|c|c|}
\hline Strains & MIC of linezolid (mg/L) & $\begin{array}{l}\text { Growth on the } \\
\text { SuperLinezolid } \\
\text { medium } \\
\text { At } 24 \mathrm{~h}\end{array}$ & At $48 \mathrm{~h}$ \\
\hline E. faecium CNR-15-307 & 32 & + & + \\
\hline S. aureus $2015 S 421$ & 8 & + & + \\
\hline S. aureus ST2015-1386 & 32 & + & + \\
\hline S. capitis ST 2015-2014 & $>256$ & + & + \\
\hline S. epidermidis ST2014-0603 & 8 & + & + \\
\hline S. epidermidis A & $>256$ & + & + \\
\hline S. epidermidis 2 & $>256$ & + & + \\
\hline S. epidermidis 22 & $>256$ & + & + \\
\hline $\begin{array}{l}\text { S. epidermidis ST } \\
2015-1734\end{array}$ & $>256$ & + & + \\
\hline $\begin{array}{l}\text { S. epidermidis ST } \\
2015-1739\end{array}$ & $>256$ & + & + \\
\hline S. epidermidis LESC & 256 & + & + \\
\hline S. epidermidis HM-1 & 16 & - & + \\
\hline S. epidermidis HM-2 & 16 & - & + \\
\hline S. epidermidis HM-3 & 256 & + & + \\
\hline S. epidermidis HM-4 & 32 & + & + \\
\hline S. epidermidis HM-5 & 256 & + & + \\
\hline S. epidermidis HM-6 & 8 & - & + \\
\hline E. faecalis ATCC 29212 & 2 & - & - \\
\hline E. faecalis N95 & 1 & - & - \\
\hline E. faecalis N89 & 1 & - & - \\
\hline E. faecalis 2953 & 2 & - & - \\
\hline E. faecalis 2094 & 2 & - & - \\
\hline E. faecalis 2146 & 2 & - & - \\
\hline E. faecalis 2147 & 2 & - & - \\
\hline E. casseliflavus 2149 & 2 & - & - \\
\hline S. aureus ATCC 29213 & 2 & - & - \\
\hline S. aureus $\mathrm{C} 1013$ & 1 & - & - \\
\hline S. aureus C1014 & 1 & - & - \\
\hline S. aureus 2954 & 2 & - & - \\
\hline S. aureus 2092 & 2 & - & - \\
\hline S. aureus 2973 & 2 & - & + \\
\hline S. aureus 3108 & 2 & - & - \\
\hline S. aureus 2959 & 2 & - & - \\
\hline S. aureus 2732 & 1 & - & - \\
\hline S. epidermidis $\mathrm{N} 30$ & 1 & - & - \\
\hline S. epidermidis N79 & 1 & - & - \\
\hline S. epidermidis 2145 & 2 & - & - \\
\hline
\end{tabular}

bacteria in each dilution, BHI agar was inoculated concomitantly with $100 \mu \mathrm{l}$ of suspension and was incubated overnight at $37^{\circ} \mathrm{C}$. The number of viable colonies was counted after $24 \mathrm{~h}$ of culture at $37^{\circ} \mathrm{C}$. The sensitivity and specificity cut-off values were set at $1 \times 10^{3} \mathrm{CFU} / \mathrm{ml}$ i.e., a limit value of $1 \times 10^{3} \mathrm{CFU} / \mathrm{ml}$ and above was considered as " not efficiently detected " (Schwarz et al., 2000).

Spiked stools were also tested using this selective culture medium, done in triplicate. Spiked fecal samples were made by adding $100 \mu \mathrm{l}$ of each strain dilution to $900 \mu \mathrm{l}$ of fecal suspension that was obtained by suspending $5 \mathrm{~g}$ of freshly pooled feces from three healthy volunteers in $50 \mathrm{ml}$ of distilled water, as done previously (Nordmann et al., 2016). A non-spiked fecal suspension was used as negative control. The lowest detection limit was determined by plating $100 \mu$ of each dilution on the screening medium. The sensitivity and specificity were determined using the same cut-off value set at $\geq 10^{3} \mathrm{CFU} / \mathrm{mL}$ (Nordmann et al., 2016).

MICs of LZD were determined using the broth microdilution method in Mueller-Hinton broth, as recommended by the CLSI (Clinical and Laboratory Standards Institute, 2018). For each strain, an inoculum corresponding to $5 \times 10^{5} \mathrm{CFU} / \mathrm{ml}$ was distributed in the 96-well tray (Sarstedt, Nümbrecht, Germany). The evaluation of the selective medium was performed in triplicate.

According to the CLSI and EUCAST breakpoints (www.eucast.org/ clinical_breakpoints/), Staphylococcus spp. and Enterococcus spp. isolates with MIC values of LZD $\leq 4 \mathrm{mg} / \mathrm{L}$ are categorized as susceptible, whereas those with MIC values $\geq 8 \mathrm{mg} / \mathrm{L}$ are categorized as resistant.

\section{Results}

Overall, all LZD-resistant Gram-positive isolates tested grew on the SuperLinezolid medium after $24 \mathrm{~h}$, except three LZD-resistant S. epidermidis isolates (HM-1, HM-2, and HM-6) that did not grow after $24 \mathrm{~h}$ of incubation (even at a concentration of $10^{3} \mathrm{CFU} / \mathrm{ml}$ ), but grew after $48 \mathrm{~h}$ (Table 2). As expected, no growth was observed for the $11 \mathrm{Gram}$-negative isolates tested.

The sensitivity was found to be $82 \%$ at $24 \mathrm{~h}$ ( 3 out of 17 isolates being missed), and reached $100 \%$ at $48 \mathrm{~h}$. At $48 \mathrm{~h}$, a single LZD-susceptible isolate grew (specificity 95\%).

For all the LZD-susceptible isolates, the lowest limit of detection was found above the cut-off value of $10^{3} \mathrm{CFU} / \mathrm{ml}$, being in fact $\geq 1 \times 10^{6} \mathrm{CFU} /$ $\mathrm{ml}$ (Table 2). By contrast, all the LZD-resistant isolates grew on the SuperLinezolid medium in $24 \mathrm{~h}$ and the lowest limit of detection was below the cut-off value. All LZD-susceptible Gram-positive did not growth after $24 \mathrm{~h}$ of incubation when the inoculum was up to $10^{3} \mathrm{CFU} / \mathrm{ml}$; small colonies were obtained only for a single isolate (S. aureus 2973) after $48 \mathrm{~h}$ of incubation when using this same inoculum. Therefore, a specificity of $100 \%$ was observed after a 24 -h culture. Finally, no Gram-negative isolate grew after either $24 \mathrm{~h}$ or $48 \mathrm{~h}$ of incubation.

The spiked stools containing LZD-resistant strains grew with a lowest detection limit ranging from $10^{1}$ to $10^{2} \mathrm{CFU} / \mathrm{ml}$ (Table 3 ), thus nicely corresponding to the required sensitivity. In addition, no false-positive isolate was recovered, thus showing an excellent specificity.

\section{Discussion}

We developed here a selective medium allowing screening and detection of Gram-positive bacteria exhibiting resistance to LZD. Considering that LZD-resistant isolates are currently emerging in different parts of the world, this selective medium may be used to perform prospective screening, and epidemiological surveys. Some surveys had used media supplemented with LZD for selection of LZD-resistant isolates but neither systematic evaluation nor development of such media had been performed (Bourgeois-Nicolaos et al., 2014; Lode et al., 2001).

The SuperLinezolid medium may detect all clinically-relevant bacterial species exhibiting resistance to LZD, regardless of the resistance

Table 3

Lowest limits of detection of the SuperLinezolid medium for a series of linezolid-resistant isolates in spiked stools.*

\begin{tabular}{|c|c|c|c|c|}
\hline Strains & Species & Genotype & MIC of linezolid $(\mu \mathrm{g} / \mathrm{ml})$ & Lowest limit of detection \\
\hline $2015 S 421$ & S. aureus & $\mathrm{Cfr}+$ & 8 & $10^{1}$ \\
\hline CNR & E. faecium & $\mathrm{CfrC}+$ OptrA + & 32 & $10^{1}$ \\
\hline ST2015-2014 & S. capitis & Mutations RNA T/2319/C; G/2576/T & $>256$ & $10^{2}$ \\
\hline ST2014-0603 & S. epidermidis & Mutations RNA T/2504/A; C/2534/T & 8 & $10^{1}$ \\
\hline LESC & S. epidermidis & ND (cfr-; optrA-) & 256 & $10^{1}$ \\
\hline $\mathrm{HM}-2$ & S. epidermidis & ND (cfr-; optrA-) & 16 & $10^{1^{*}}$ \\
\hline HM-3 & S. epidermidis & $\mathrm{Cfr}+$ & 256 & $10^{1}$ \\
\hline HM-6 & S. epidermidis & $\mathrm{Cfr}+$ & 8 & $10^{2}$ \\
\hline
\end{tabular}

* Only after $48 \mathrm{~h}$ growth. 
mechanism, and regardless of their resistance level to LZD. Growth of most isolates showing MICs of LZD ranging from 8 to $>256 \mu \mathrm{g} / \mathrm{ml}$ on this selective medium was confirmed. These resistant isolates were either S. aureus, S. epidermidis, E. faecium, or S. capitis. Failure to detect any LZDresistant isolate tested was observed in three cases after a 24-h culture, corresponding to S. epidermidis isolates having an MIC at 8 or $16 \mu \mathrm{g} / \mathrm{ml}$, therefore close to the breakpoint. The sensitivity was improved and all isolates detected at $48 \mathrm{~h}$; however, the specificity at $48 \mathrm{~h}$ was lower, with 6 out of 20 LZD-susceptible isolates being recovered. It seems therefore that a 24-h growth is recommended. An extended evaluation of this selective medium is needed to guarantee high specificity and high sensitivity of the selection. We may admit that our study was performed with a limited number of isolates (due to limited isolates available in our collection), nevertheless we believe that the performances of the SuperLinezolid medium as obtained here do have a significant value.

This medium offers the possibility either to assess the LZD resistance feature by selecting such isolates after re-striking of mixed cultures, but most of all to select for those LZD-resistant isolates from stools in a context of direct screening.

Therefore, the use of this selective medium may contribute to rapidly identify carriers of LZD-resistant isolates, and consequently to rapidly implement infection control measures in order to limit their spread in hospital settings.

Finally, this medium may be also useful for screening food-producing - animal reservoirs. Indeed, resistance to LZD in Enterococci has been increasingly identified particularly in swine, poultry and cattle, mainly in China (Fang et al., 2018). An animal reservoir of LZD-resistant strains has been demonstrated for a series of bacterial species, including E. faecalis and E. faecium (pigs and chicken), but also for Enterococcus gallinarum, Enterococcus casseliflavus, Enterococcus thailandicus, and Enterococcus suis (pigs) (Sadowy, 2018). Prospective surveys may therefore be conducted by veterinary networks in order to evaluate the rate of LZDresistant Gram positives in animal husbandries, and identify possible reservoirs.

\section{Funding}

This work was financed by the University of Fribourg, Switzerland. It has also been funded by the Swiss National Science Foundation (project FNS-407240_177382). A.R.-V. was partly financed by a grant from the Spanish Society of Infectious Diseases and Clinical Microbiology (SEIMC).

\section{Conflict of interest}

None to declare.

\section{Acknowledgments}

We are grateful to Prof. V. Cattoir (Rennes, France), Prof. F. Laurent (Lyon, France), and Dr. J.-W. Decousser (Créteil, France) who provided a series of well-characterized LZD resistant isolates. This work has been founded by the University of Fribourg, Switzerland and by the NARA (Switzerland).

\section{References}

Antonelli A, D'Andrea MM, Brenciani A, Galeotti CL, Morroni G, Pollini S, et al. Characterization of poxtA, a novel phenicol- oxazolidinone-tetracycline resistance gene from an MRSA of clinical origin. J Antimicrob Chemother 2018;73(7):1763-9. https://doi.org/ 10.1093/jac/dky088.

Bi R, Qin T, Fan W, Ma P, Gu B. The emerging problem of linezolid-resistant enterococci. J Glob Antimicrob Resist 2017;13:11-9.

Bourgeois-Nicolaos N, Nguyen TT, Defrance G, Massias L, Alavoine L, Lefort A, et al. The emergence of linezolid resistance among enterococci in intestinal microbiota of treated patients is unrelated to individual pharmacokinetic characteristics. Antimicrob Agents Chemother 2014;58:2681-7.

Cattoir V, Giard JC. Antibiotic resistance in Enterococcus faecium clinical isolates. Expert Rev Anti Infect Ther 2014:12:239-48.

Clinical and Laboratory Standards Institute (CLSI). Performance standards for antimicrobial susceptibility testing: Twenty-eighth informational supplement M100-S28. Wayne, PA, USA: CLSI; 2018.

Fang LX, Duan JH, Chen MY, Deng H, Liang HQ, Xiong YQ et al. Prevalence of cfr in Enterococcus faecalis strains isolated from swine farms in China: predominated cfr-carrying pCPPF5-like plasmids conferring "non-linezolid resistance" phenotype. Infect Genet Evol 2018;62:188-92.

Gu B, Kelesidis T, Tsiodras S, Hindler J, Humphries RM. The emerging problem of linezolidresistant Staphylococcus. J Antimicrob Chemother 2013;68:4-11.

Kehrenberg C, Schwarz S, Jacobsen L, Hansen LH, Vester B. A new mechanism for chloramphenicol, florfenicol and clindamycin resistance: methylation of $23 \mathrm{~S}$ ribosomal RNA at A2503. Mol Microbiol 2005;57:1064-73.

Leach KL, Brickner SJ, Noe MC, Miller PF. Linezolid, the first oxazolidinone antibacterial agent. Ann N Y Acad Sci 2011:1222:49-54.

Lode H, Von der Höh N, Ziege S, Borner K, Nord CE. Ecological effects of linezolid versus amoxicillin/clavulanic acid on the normal intestinal microflora. Scand J Infect Dis 2001;33:899-903.

Long KS, Poehlsgaard J, Kehrenberg C, Schwarz S, Vester B. The Cfr rRNA methyltransferase confers resistance to phenicols, lincosamides, oxazolidinones, pleuromutilins, and streptogramin a antibiotics. Antimicrob Agents Chemother 2006;50:2500-5.

Marín M, Martín A, Alcalá L, Cercenado E, Iglesias C, Reigadas E, et al. Clostridium difficile isolates with high linezolid MICs harbor the multiresistance gene cfr. Antimicrob Agents Chemother 2015;59:586-9.

Nordmann P, Girlich D, Poirel L. Detection of carbapenemase producers in Enterobacteriaceae by use of a novel screening medium. J Clin Microbiol 2012;50:2761-6.

Nordmann P, Jayol A, Poirel L. A universal culture medium for screening polymyxinresistant gram-negative isolates. J Clin Microbiol 2016;54:1395-9.

Pfaller MA, Mendes RE, Streit JM, Hogan PA, Flamm RK. Five-year summary of in vitro activity and resistance mechanisms of linezolid against clinically important gram-positive cocci in the United States from the LEADER surveillance program (2011 to 2015). Antimicrob Agents Chemother 2017a;61. [pii:e00609-17].

Pfaller MA, Mendes RE, Streit JM, Hogan PA, Flamm RK. ZAAPS program results for 2015 an activity and spectrum analysis of linezolid using clinical isolates from medical centres in 32 countries. J Antimicrob Chemother 2017b;72:3093-9.

Poirel L, Jayol A, Nordmann P. Polymyxins: antibacterial activity, susceptibility testing, and resistance mechanisms encoded by plasmids or chromosomes. Clin Microbiol Rev 2017:30:557-96.

Purrello SM, Garau J, Giamarellos E, Mazzei T, Pea F, Soriano A, et al. Methicillin-resistant Staphylococcus aureus infections: a review of the currently available treatment options. J Glob Antimicrob Resist 2016;7:178-86.

Sadowy E. Linezolid resistance genes and genetic elements enhancing their dissemination in enterococci and streptococci. Plasmid 2018;99:89-98.

Schwarz S, Werckenthin C, Kehrenberg C. Identification of a plasmid-borne chloramphenicol-florfenicol resistance gene in Staphylococcus sciuri. Antimicrob Agents Chemother 2000;44:2530-3.

Sharkey LK, Edwards TA, O'Neill AJ. ABC-F proteins mediate antibiotic resistance through ribosomal protection. MBio 2016;7, e01975.

Tang Y, Dai L, Sahin O, Wu Z, Liu M, Zhang Q. Emergence of a plasmid-borne multidrug resistance gene $c f r(C)$ in foodborne pathogen Campylobacter. J Antimicrob Chemother 2017:72:1581-8.

Wang Y, Lv Y, Cai J, Schwarz S, Cui L, Hu Z, et al A novel gene, optrA, that confers transferable resistance to ox- azolidinones and phenicols and its presence in Enterococcus faecalis and Enterococcus faecium of human and animal origin. J Antimicrob Chemother 2015;70:2182-90.

Zahedi Bialvaei A, Rahbar M, Yousefi M, Asgharzadeh M, Samadi Kafil H. Linezolid: a promising option in the treatment of gram-positives. J Antimicrob Chemother 2017;72:354-64. 